\title{
Agriculture pest and disease risk maps considering MSG satellite data and Land Surface Temperature
}

\author{
J. R. Marques da Silva ${ }^{(1, a b c *)}$, Carlos Viegas Damásio ${ }^{(2)}$, A. M. O. Sousa ${ }^{(1, a b)}$, L. Bugalho ${ }^{(3)}$, \\ L. Pessanha ${ }^{(3)}$, P. Quaresma ${ }^{(1, b)}$ \\ ${ }^{(1)}$ Universidade de Évora, Escola de Ciências e Tecnologia, Departamento de Engenharia Rural. P. O. BOX. \\ 94, 7002 - 554 Évora, Portugal, Tel: (+351) 266760823, Fax: (+351) 266711189. *E-mail: jmsilva@uevora.pt; \\ ${ }^{(a)}$ ICAAM - Instituto de Ciências Agrárias e Ambientais Mediterrânicas, ${ }^{(b)}$ CITI - Centro de Inovação em \\ Tecnologias de Informação, Évora, Portugal, ${ }^{(c)}$ Applied Management and Space Centre for Interdisciplinary \\ Development and Research on Environment (DREAMS), Lisbon, Portugal. \\ ${ }^{(2)}$ Universidade Nova de Lisboa - Faculdade de Ciências e Tecnologia. NOVALINCS and CENTRIA. \\ Departamento de Informática da FCT/UNL. Quinta da Torre 2829-516 Caparica, Portugal. \\ ${ }^{(3)}$ Instituto Português do Mar e da Atmosfera (IPMA), Rua C do Aeroporto, 1749-077 Lisboa, Portugal \\ Tel: (+351) 218447 000, Fax: (+351) 218402370
}

\begin{abstract}
Pest risk maps for agricultural use are usually constructed from data obtained from in-situ meteorological weather stations, which are relatively sparsely distributed and are often quite expensive to install and difficult to maintain. This leads to the creation of maps with relatively low spatial resolution, which are very much dependent on interpolation methodologies. Considering that agricultural applications typically require a more detailed scale analysis than has traditionally been available, remote sensing technology can offer better monitoring at increasing spatial and temporal resolutions, thereby improving pest management results and
\end{abstract}


reducing costs. This article uses ground temperature, or land surface temperature (LST), data distributed by EUMETSAT/LSASAF (with a spatial resolution of $3 \times 3 \mathrm{~km}$ (nadir resolution) and a revisiting time of $15 \mathrm{~min}$ ) to generate one of the most commonly used parameters in pest modelling and monitoring: "thermal integral over air temperature (accumulated degree-days)". The results show a clear association between the accumulated LST values over a threshold and the accumulated values computed from meteorological stations over the same threshold (specific to a particular tomato pest). The results are very promising and enable the production of risk maps for agricultural pests with a degree of spatial and temporal detail that is difficult to achieve using in-situ meteorological stations.

Keywords: Land Surface Temperature, LST, Satellite Application Facility, SAF, EUMETSAT, MSG, Pest Management, Pest risk maps. 